\section{Efeitos de diferentes intensidades de treinamento aeróbio sobre a composição corporal em adolescentes obesos}

\section{Effects of different intensities of aerobic training on body composition in obese adolescents}

Priscyla Praxedes Gomes ${ }^{1}$

Humberto José Gomes da Silva ${ }^{1}$

Camila Tenório Calazans de Lira ${ }^{1}$

Mara Cristina Lofrano-Prado ${ }^{2}$

Wagner Luiz do Prado ${ }^{1}$

Resumo - O objetivo do presente estudo foi verificar os efeitos de diferentes intensidades de treinamento físico aeróbio sobre a composição corporal de adolescentes obesos submetidos à intervenção multidisciplinar. O estudo caracterizou-se como ensaio clínico aleatorizado. Quarenta e dois adolescentes ( 13 a 17 anos) obesos (IMC $=34,48 \pm 3,88 \mathrm{~kg} / \mathrm{m}^{2}$ ) púberes (estágio 3 e 4) participaram de 12 semanas de intervenção multidisciplinar. Os adolescentes foram alocados aleatoriamente em dois grupos experimentais: treinamento de alta intensidade (TAI) $(\mathrm{n}=20)$ - intensidade correspondente ao limiar ventilatório I (LVI); e treinamento de baixa intensidade (TBI) $(n=22)$ - $20 \%$ abaixo do LVI. As sessões de exercício foram isocalóricas $(350 \mathrm{kcal})$. Todos os voluntários receberam acompanhamento clínico, nutricional e psicológico. A massa corporal $(\mathrm{kg})$, estatura $(\mathrm{cm})$ e dobras cutâneas $(\mathrm{mm})$ foram mensuradas. O percentual de gordura foi estimado por meio da equação de Slaughter, em seguida, a massa magra e massa gorda foram estimadas. Após 12 semanas de intervenção foram verificadas reduções na massa corporal, IMC e adiposidade em ambos os grupos $(\mathrm{p}<0,001)$, bem como elevação da massa magra dos adolescentes $(\mathrm{p}<0,001)$. No presente estudo, a intervenção multidisciplinar se mostrou efetiva na modulação da composição corporal e da adiposidade dos adolescentes obesos. Entretanto, tais resultados parecem ser independentes da intensidade de treinamento aeróbio a qual os grupos foram submetidos.

Palavras-chave: Composição corporal; Obesidade; Treinamento aeróbio.

Abstract - This randomized clinical trial aimed to verify the effects of different aerobic training intensities on body composition in obese adolescents submitted to a 12-week multidisciplinary intervention. Participants were 42 obese and pubscent adolescents (BMI $=34.48$ $\pm 3.88 \mathrm{~kg} / \mathrm{m} 2$; aged 13-17 years). The adolescents were randomized into two experimental groups: high intensity training (HIT) $(n=20)$ - corresponding to the ventilatory threshold I (LVI); and low intensity training (LIT) $(n=22)-20 \%$ below LVI. Exercise sessions were isocaloric ( $350 \mathrm{kcal}$ ). All participants received nutritional, psychological and clinical follow-up. Body mass ( $\mathrm{kg}$ ), height ( $\mathrm{cm}$ ) and skinfold thickness ( $\mathrm{mm}$ ) were measured. Body fat (\%) was estimated using the Slaughter equation, followed by the calculation of lean mass and fat mass. After the 12-week intervention, we observed a decrease in body weight, BMI and body fat in both groups $(p<0.001)$, as well as an increase in lean body mass of the adolescents $(p<0.001)$. In this study, the multidisciplinary intervention was effective to modulate body composition and adiposity among obese adolescents. However, the results seem to be independent of aerobic training intensities.

Key words: Aerobic training; Body composition; Obesity.
1 Universidade de Pernambuco. Escola Superior de Educação Física. Recife, PE. Brasil.

2 Universidade de Pernambuco. Grupo de Estudos em Nutrição e Exercício. Recife, PE. Brasil.

Recebido em 16/08/12 Revisado em 03/10/12 Aprovado em 24/02/13 


\section{INTRODUÇÃO}

A obesidade é uma doença crônica caracterizada pelo acúmulo excessivo de gordura corporal em decorrência de um balanço energético positivo crônico ${ }^{1}$, sua etiologia está associada à redução dos níveis de atividade física e ingestão de alimentos de alta densidade energética ${ }^{2}$. No Brasil, a obesidade acomete cerca de $14 \%$ dos adolescentes, e ao contrário do observado em décadas anteriores, atualmente, verifica-se uma manutenção da prevalência de obesidade ${ }^{3}$.

O excesso de adiposidade é responsável pelo aumento na concentração circulante de diferentes marcadores inflamatórios, especialmente, os produzidos pelo tecido adiposo, denominados de adipocina ${ }^{4}$. Este estado de inflamação crônica subclínica verificado em obesos, está diretamente associado a várias desordens metabólicas, tais como, resistência à ação da insulina, diminuição da tolerância à glicose, hipertrigliceridemia, hipercolesterolemia com concomitante redução da lipoproteína de alta densidade (HDL) e alterações nos níveis pressóricos ${ }^{5}$.

Desta forma, o monitoramento da composição corporal durante a puberdade é de essencial importância, visto que, durante este período do desenvolvimento, a composição corporal é um potente preditor da massa corporal na fase adulta, adicionalmente, alguns aspectos da composição corporal, durante a puberdade, são fatores de risco para o desenvolvimento de doenças cardiovasculares, diabetes mellitus e osteoporose. Com isso, torna-se importante o tratamento precoce da obesidade, evitando que esses adolescentes se tornem adultos obesos ${ }^{6}$. Neste sentido, postula-se que programas multidisciplinares compostos por orientação nutricional, exercício físico e acompanhamento psicológico sejam os mais indicados para o tratamento da obesidade, sendo mais efetivos em crianças e adolescentes do que em adultos ${ }^{6,7}$.

O exercício físico, independente da intensidade que é realizado, é eficiente na redução da adiposidade corporal, entretanto, estudos prévios não apresentam uma padronização quanto à prescrição da intensidade do esforço (percentual da frequência cardíaca, consumo máximo de oxigênio ou ainda a velocidade aeróbica máxima), o que dificulta a comparação entre os resultados ${ }^{8,9}$. Portanto, o objetivo do presente estudo foi verificar os efeitos de diferentes intensidades de treinamento aeróbio sobre a composição corporal de adolescentes obesos submetidos à intervenção multidisciplinar.

\section{PROCEDIMENTOS METODOLÓGICOS}

Este estudo caracteriza-se como ensaio clínico aleatorizado com delineamento longitudinal. O estudo foi aprovado pelo Comitê de Ética da Universidade de Pernambuco (154/09). Todos os responsáveis assinaram o termo de consentimento livre e esclarecido.

Após divulgação em rádio, televisão e jornais locais, 229 adolescentes de ambos os gêneros se voluntariaram para participar do estudo, 
entretanto 187 não atenderam aos critérios inclusão: Ter idade entre 13 e 17 anos, massa corporal até $120 \mathrm{~kg}$, índice de massa corporal (IMC) acima do percentil 95, de acordo com os critérios do Centers for Disease Control and Prevention (CDC) ${ }^{10}$, estágio maturacional púbere (estágio 3 e 4) de acordo com os critérios de Tanner $(1976)^{11}$ e motivação intrínseca para participar de um programa multidisciplinar em longo prazo (avaliada por uma psicóloga). Ao final, quarenta e dois voluntários (13 meninos e 29 meninas) atenderam aos critérios e foram incluídos no estudo. Após as avaliações basais, os participantes foram aleatoriamente (utilizando-se o método coinfliping) alocados no grupo treinamento de alta intensidade (TAI) $(n=20)$ ou grupo treinamento de baixa intensidade (TBI) $(\mathrm{N}=22)$. Todos os adolescentes receberam a mesma intervenção nutricional, psicológica e clínica. Os critérios de exclusão adotados foram gravidez durante o tratamento e frequência inferior a $75 \%$ a qualquer uma das intervenções (física, nutricional, clínica ou psicológica) (Figura 1).

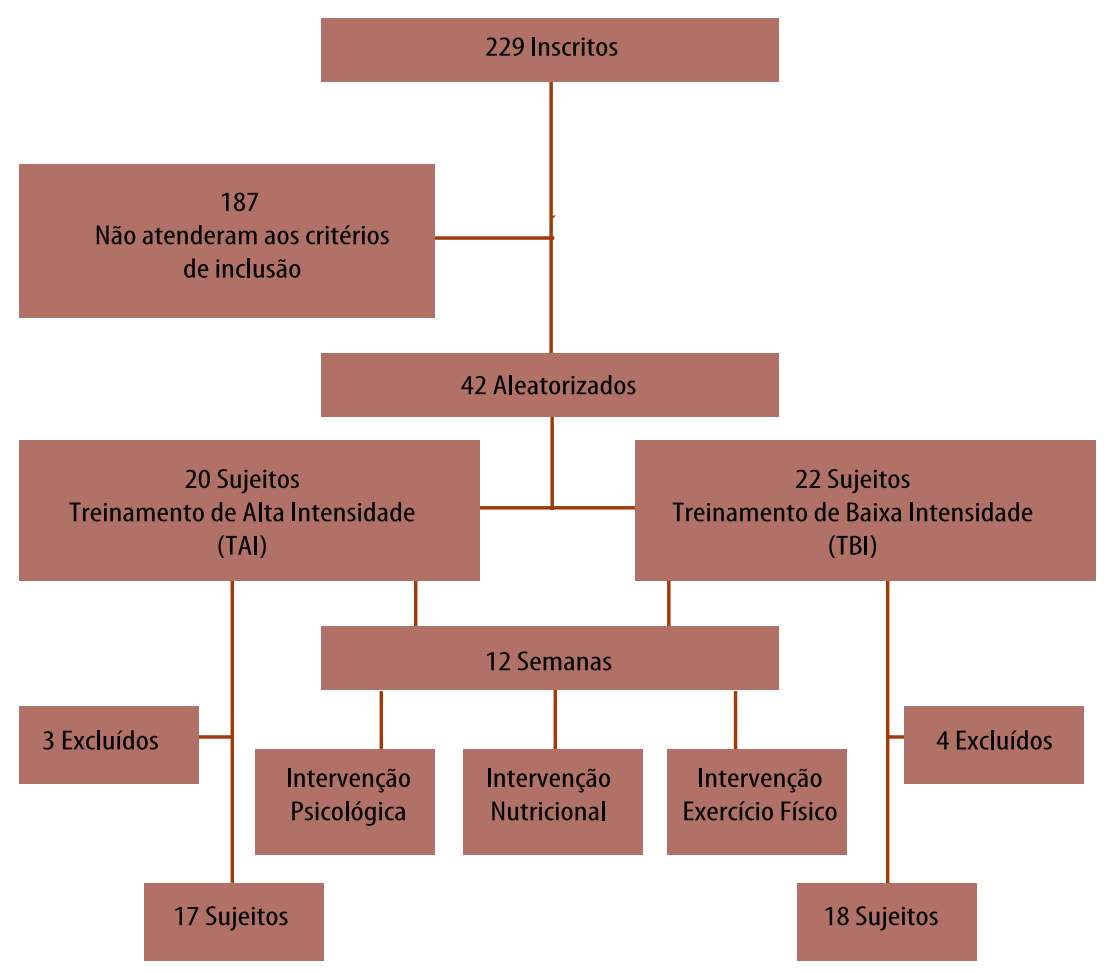

Figura 1. Desenho Experimental.

\section{Intervenção Clínica}

A partir da primeira consulta com a endocrinologista responsável pelo projeto, os adolescentes receberam acompanhamento clínico mensal. Durante as consultas recebiam orientações sobre mudanças no estilo de vida e acompanhamento da evolução clínica dos voluntários. Não houve prescrição de nenhum tipo de medicação. 


\section{Intervenção Nutricional}

A intervenção nutricional foi composta por encontros em grupos (aproximadamente 09 adolescentes), uma vez por semana, com duração de uma hora. As atividades sempre foram desenvolvidas e supervisionadas pela nutricionista do grupo. Durante os encontros, eram abordados temas como pirâmide alimentar, fastfood, rotulagem nutricional, dietas da moda, nutrição na promoção de saúde, tipos de gordura (trans, saturada e insaturada), produtos diet e light, comportamento em ocasiões especiais (festas, aniversários, eventos sociais). Não foi prescrita nenhuma dieta individualizada.

\section{Intervenção Psicológica}

Foram realizados atendimentos psicológicos em grupos (aproximadamente 09 adolescentes), uma vez por semana, durante 1 hora, conduzidos pela psicóloga do grupo. Nessas sessões, foram realizadas dinâmicas para se abordar temas como emoções (sentimentos), autoestima, imagem corporal, preconceito, transtornos alimentares, dificuldades, questões familiares, bem como questões que pudessem surgir no grupo. Além disso, a psicóloga ficava à disposição dos voluntários para apoio e acolhimento, se necessário.

\section{Treinamento Aeróbio}

Os participantes de ambos os grupos (TAI e TBI) realizaram treinamento aeróbio individualizado em esteira rolante, três vezes por semanas, sob a supervisão de um profissional de Educação Física. Os voluntários do TAI treinaram em intensidade correspondente ao limiar ventilatório I (LVI), e os do TBI exercitaram-se em intensidade $20 \%$ abaixo do LVI. Para ambos os grupos, as sessões foram isocalóricas, com o gasto energético fixado em $350 \mathrm{kcal} / \mathrm{sessão,} \mathrm{estimado} \mathrm{por} \mathrm{calorimetria} \mathrm{indireta.} \mathrm{Uma} \mathrm{vez} \mathrm{que} \mathrm{cada}$ voluntário treinava em uma carga específica, a duração das sessões diferiu entre os sujeitos, para garantir que o gasto energético pré-fixado fosse atingido; a duração de cada sessão foi determinada considerando-se que para cada litro de oxigênio consumido $\left(\mathrm{VO}_{2}\right), 4,96 \mathrm{kcal}$ de energia era liberado, baseado no equivalente metabólico da tarefa (MET), conforme a seguir:

$$
\text { Duração da sessão de exercício (min.) = } 350 \text { kcal / (V0 na carga*1MET) }
$$

\section{Mensuração}

Todos os voluntários foram submetidos ao mesmo protocolo de avaliações, antes (pré) e após 12 semanas (pós) de intervenção multidisciplinar. As medidas eram realizadas sempre no mesmo período do dia, com o objetivo de se minimizar quaisquer variações circadianas nas variáveis analisadas.

\section{Medidas Antropométricas e Composição Corporal}

As medidas de massa corporal (MC), estatura e circunferência de cintura (CC) foram realizadas em uma balança Filizola ${ }^{\circledR}$ com precisão de $0,1 \mathrm{~kg}$, um estadiômetro de madeira com precisão de $0,1 \mathrm{~cm}$ e fita métrica inex- 
tensível com precisão de 0,1 cm. Em seguida, foi calculado o índice de massa corporal (IMC), dividindo-se a massa corporal ( $\mathrm{kg}$ ) pelo quadrado da estatura $\left(\mathrm{m}^{2}\right)$.

As dobras cutâneas tricipital (TR) e subescapular (SE) foram mensuradas com um adipômetro de marca Lange $e^{\circledast}$ com resolução de $1 \mathrm{~mm}$. A dobra cutânea TR, foi medida na face posterior do braço direito, no sentido longitudinal, no ponto médio do processo acromial da escápula e o processo olecraniano da ulna. A dobra cutânea SE seguiu o sentido diagonal (45) do ângulo inferior da escapula direita. A padronização seguiu as recomendações propostas por Petroski et al. ${ }^{12}$. Duas medidas foram realizadas em forma de circuito, em seguida, a média foi calculada. $\mathrm{O}$ percentual de gordura $(\% \mathrm{G})$ foi estimado por meio da equação de Slaughter et al. ${ }^{13}$, específica para cada gênero.

\section{Consumo de Oxigênio e Determinação do Limiar Ventilatório}

$\mathrm{O} \mathrm{VO}_{2}$ foi determinado por meio de análise direta de gases, utilizando-se um analisador metabólico de circuito aberto (Cortex Biophysik Metalyzer IIB, Germany), durante teste contínuo e incremental em esteira rolante (Inbrasport Super ATL, Brazil). Após aquecimento de 3 minutos a $3 \mathrm{~km} / \mathrm{h}$, a velocidade era acrescida em $1 \mathrm{~km} / \mathrm{h}$ a cada minuto, até a exaustão voluntária, ou quando a escala de Borg e o quociente respiratório apresentassem valores superiores a 18 e 1,15, respectivamente. A inclinação foi mantida constante em $1 \%$ durante todo o teste. O maior $\mathrm{VO}_{2}$ obtido antes da interrupção do teste foi considerado como consumo de pico de oxigênio $\left(\mathrm{VO}_{2 \text { pico }}\right)$. Antes de cada teste, os instrumentos eram calibrados para a mistura de gases $\left(\mathrm{O}_{2}\right.$ $=12,2$ e $\mathrm{CO}_{2}=4,8$ - White Martins) e volume (3L CardioequipoSyringe, Brazil), seguindo-se todas as instruções do fabricante.

O LVI foi determinado por meio de inspeção visual por dois pesquisadores independentes, como o ponto de quebra de linearidade entre a produção de dióxido de carbono $\left(\mathrm{CO}_{2}\right)$ e o $\mathrm{VO}_{2}(\mathrm{~V}$-slope); ponto no qual a curva do equivalente ventilatório de oxigênio $\left(\mathrm{VE} / \mathrm{VO}_{2}\right)$ e a fração expirada de $\mathrm{CO}_{2}\left(\mathrm{P}_{\mathrm{ET}} \mathrm{CO}_{2}\right)$ atingiram seus menores valores, imediatamente antes do aumento dos respectivos equivalentes ${ }^{14}$.

\section{Análise Estatística}

Os dados foram submetidos ao teste de normalidade de Shapiro Wilk. ANOVA two-way para medidas repetidas com post hoc de Fisher foi adotada para verificar possíveis efeitos entre os grupos (TBI e TAI) e momentos (pré e pós). Os valores são apresentados em média \pm DP e delta de variação percentual [ $\Delta \%=$ (medida pós-medida pré)/medida pré $\left.\left.{ }^{\star} 100\right)\right]$. A análise estatística foi realizada utilizando-se o Software Statistica 7. O nível de significância foi fixado em $\mathrm{p}<0,05$. O delineamento amostral foi baseado em estudos prévios, considerando-se um erro estimado de 0,40, erro alfa de 0,05 e beta de 0,80. Desta forma, a amostra mínima necessária foi determinada em 16 voluntários por grupo. 


\section{RESULTADOS}

A amostra foi composta por 42 adolescentes (13 meninos e 29 meninas; $15,4 \pm 1,5$ anos; $34,4 \pm 4,3 \mathrm{~kg} / \mathrm{m}^{2}$ ), entretanto, durante a intervenção, sete voluntários foram excluídos, dos quais, três do TAI (um por problemas ortopédicos e dois por aderência inferior a 75\%) e quatro do TBI (aderência inferior a 75\%). Após 12 semanas de tratamento, verificou-se uma adesão de $85 \%$ para o TAI (06 meninos e 11 meninas) e de $81 \%$ ( 11 meninas e 07 meninos) para o TBI, sem diferenças entre os grupos. As sessões de TBI foram mais longas $(57,1 \pm 11,71 \mathrm{~min})$ do que TAI $(40,7 \pm 8,71 \mathrm{~min})(\mathrm{p}<0,000)$, sem diferenças no gasto energético (350 kcal).

As características antropométricas e da composição corporal dos voluntários são apresentadas na Tabela 1. Após 12 semanas de intervenção, foram verificadas reduções na massa corporal, IMC e adiposidade, bem como elevação da massa magra em ambos os grupos ( $\mathrm{p}<0,001)$. A variação percentual da massa gorda foi de $-27,31 \%$ para o TBI e de $-20,57 \%$ para o TAI (figura 2), e de massa magra de $30,3 \%$ no TBI e $16,3 \%$ no TAI (figura 2). Vale ressaltar que, em nenhum dos momentos analisados foram verificadas diferenças entre os grupos experimentais.

Tabela 1. Efeitos de diferentes intensidades de treinamento aeróbio sobre variáveis antropométricas e composição corporal em adolescentes obesos submetidos à intervenção multidisciplinar

\begin{tabular}{lcccc}
\hline & \multicolumn{2}{c}{ TBI $(\mathrm{n}=18)$} & \multicolumn{2}{c}{ TAI $(\mathrm{n}=17)$} \\
\hline & Pré & Pós & Pré & Pós \\
\hline Idade (anos) & $15,34 \pm 1,63$ & $15,66 \pm 1,63$ & $15,77 \pm 1,34$ & $16,10 \pm 1,34$ \\
\hline Estatura $(\mathrm{m})$ & $1,62 \pm 0,08$ & $1,63 \pm 0,09$ & $1,62 \pm 0,05$ & $1,62 \pm 0,05$ \\
MC $(\mathrm{kg})$ & $92,1 \pm 11,4$ & $90,4 \pm 12,3^{*}$ & $90,3 \pm 11,2$ & $87,7 \pm 11,7^{*}$ \\
\hline IMC $\left(\mathrm{kg} / \mathrm{m}^{2}\right)$ & $34,6 \pm 3,7$ & $33,7 \pm 3,9^{*}$ & $34,3 \pm 4,1$ & $33,4 \pm 4,0^{*}$ \\
\%G & $53,1 \pm 9,3$ & $38,0 \pm 7,0^{*}$ & $49,9 \pm 6,9$ & $40,4 \pm 9,7^{*}$ \\
MG $(\mathrm{kg})$ & $50,1 \pm 13,8$ & $37.6 \pm 9,6^{*}$ & $46,3 \pm 9,3$ & $40,0 \pm 13,0^{*}$ \\
MM (kg) & $43,1 \pm 6,3$ & $55,2 \pm 9,2^{*}$ & $50,0 \pm 6,9$ & $59,5 \pm 9,7^{*}$ \\
\hline
\end{tabular}

$M C=$ massa corporal; $I M C=$ Índice de Massa Corporal; $\% G=$ percentual de gordura; $M G=$ massa gorda; $\mathrm{MM}=$ massa magra. ${ }^{*} \mathrm{p}<0,05$ pré vs pós; $\mathrm{TBI}=$ Treinamento de Baixa Intensidade; $\mathrm{TAl}=$ Treinamento de Alta Intensidade.
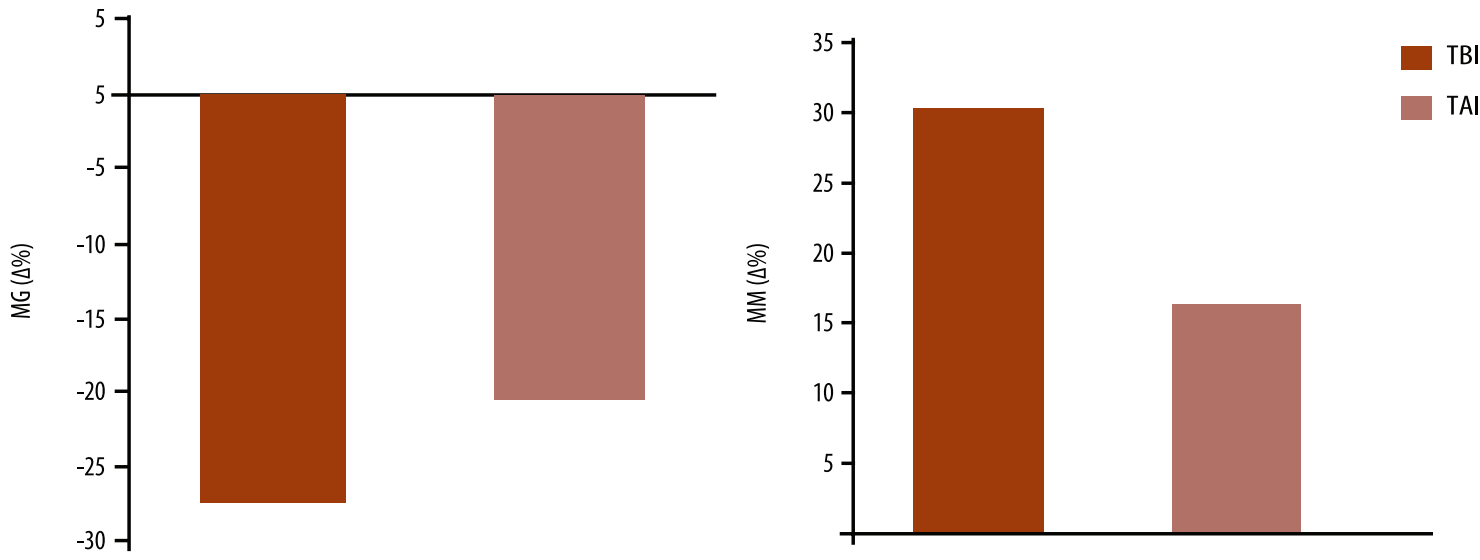

Figura 2. Efeito da intervenção multidisciplinar após 12 semanas sobre a (a) massa gorda e (b) massa magra em adolescentes obesos. MG= Massa Gorda; $\mathrm{MM}=$ Massa Magra. $\mathrm{TBI}=$ Treinamento em Baixa Intensidade; TAI= Treinamento em Alta Intensidade. 


\section{DISCUSSÃO}

Estudos relacionados à composição corporal são importantes no tratamento e controle da obesidade, visto que o excesso de gordura corporal tem associação com diferentes doenças metabólicas (dislipidemia, resistência à ação da insulina, diabetes mellitus) e crônicas não transmissíveis (hipertensão arterial, Insuficiência renal, arteriosclerose $)^{15,16}$. Desta forma, o principal achado do presente estudo é que tanto o treinamento aeróbio de alta intensidade quanto o de baixa intensidade promovem alterações benéficas na composição corporal de adolescentes obesos.

Assim como no presente estudo, Lazzer et al. ${ }^{17}$, após submeteram 24 adolescentes obesos a três semanas de treinamento aeróbio, em intensidades correspondentes a $40 \%$ e $70 \%$ do $\mathrm{VO}_{2 \max }$, com gasto energético fixado em $20 \mathrm{~kJ} / \mathrm{kg}$ de massa magra, verificaram redução na massa gorda em ambos os grupos, sem diferenças entre as intensidades, resultado semelhante foi verificado, também, em adultos com sobrepeso submetidos a três semanas de treinamento aeróbio de alta intensidade e baixa duração, e moderada intensidade e moderada duração, com um gasto fixado em $23 \mathrm{kcal} / \mathrm{kg}^{18}$.

Em condições metabólicas normais, um estado de balanço energético negativo crônico induzido por redução na ingestão alimentar ou elevação do gasto energético, acarreta em redução da massa corporal ${ }^{19}$, e quanto maior o déficit energético maior a redução na adiposidade corporal ${ }^{20}$, ou seja, em situações em que o déficit calórico for igual, as alterações na composição corporal serão semelhantes, independente da intensidade do exercício.

Indivíduos adultos quando submetidos a intervenções para redução ponderal, geralmente, apresentam redução de massa adiposa com concomitante redução também de massa magra ${ }^{21,22}$, entretanto, em adolescentes, a redução da adiposidade corporal, advinda de intervenções com déficit energético moderado (<1.000kcal/dia), nem sempre, vem acompanhada por redução na massa magra ${ }^{23,24}$.

Neste sentido, um estudo realizado por Prado et al. ${ }^{15}$, com o objetivo de verificar os efeitos da terapia multidisciplinar em longo prazo ( 12 e 24 semanas) sobre o comportamento alimentar de 62 adolescentes obesos, verificou um aumento da massa magra (em meninos e meninas) após 12 e 24 semanas de treinamento aeróbio moderado (50\% a 70\% do consumo de máximo de oxigênio). Neste mesmo sentido, Stella et al. ${ }^{25}$ reportaram aumento da massa magra em adolescentes obesas submetidas a treinamento aeróbio de alta intensidade. Vale ressaltar que tais estudos utilizaram métodos precisos para estimava da composição corporal, como o DEXA e plestimografia.

Uma vez que a adolescência é uma fase altamente anabólica, devido especialmente à grande concentração circulante de hormônios anabólicos, tais como hormônio do crescimento $(\mathrm{GH})$ e testosterona ${ }^{26}$, dessa forma, especulamos que as sessões de exercício, mesmo com predominância ae- 
róbia, tenham ocasionado estímulo suficiente para ocasionar hipertrofia muscular nos adolescentes. Infelizmente, não existem estudos na literatura que possam suportar esta hipótese, sendo necessários estudos futuros que possam comprovar, ou não, nossa hipótese. Entretanto, no presente estudo, não houve controle da ingestão alimentar dos adolescentes, o que pode ter influenciado na grande magnitude de elevação da massa magra quando comparada aos demais estudos.

Vale ressaltar que, mesmo os adolescentes permanecendo obesos, após as 12 semanas de intervenção, as modificações na composição corporal são clinicamente relevantes, visto que mesmo pequenas modificações na massa corporal são eficientes para o controle da pressão arterial sistólica e diastólica, colesterol total e triglicérides ${ }^{27}$, adicionalmente, a elevação da massa magra está associada à elevação da taxa metabólica de repouso ${ }^{28}$, o que pode ser considerado como um preditor da manutenção da massa corporal em longo prazo ${ }^{29}$.

Uma das limitações do nosso estudo foi à ausência de medidas mais precisas da composição corporal (DEXA), visto que, a literatura indica o DEXA como uma ferramenta mais eficaz para estimar o percentual de gordura para indivíduos obesos, portanto, as interpretações não podem ser generalizadas. Tornando-se importantes futuros estudos com dados mais precisos para a determinada amostra.

Por outro lado, o ponto forte do presente estudo é que as intensidades de treinamento foram baseadas no limiar anaeróbio, que é considerado, hoje em dia, a melhor variável para prescrição e controle da sobrecarga metabólica, a qual o organismo é submetido, minimizando diferenças individuais, comumente observadas quanto à intensidade do esforço é relativa pelo $\mathrm{VO}_{2 \max }{ }^{30}$.

Por fim, vale ressaltar que, uma vez que os adolescentes foram submetidos à intervenção multidisciplinar, os resultados aqui reportados não podem ser atribuídos exclusivamente ao treinamento aeróbio, as contribuições das demais intervenções devem ser consideradas na interpretação dos dados.

\section{CONCLUSÃO}

Os resultados do presente estudo demonstraram que, o treinamento aeróbio, independente da intensidade em que é realizado, é capaz de promover alterações positivas na composição corporal de adolescentes obesos submetidos à intervenção multidisciplinar, não apenas reduzindo a adiposidade corporal, mas também auxiliando na manutenção e elevação da massa magra nesta população.

\section{Agradecimentos:}

Os autores agradecem ao CNPq e a FACEPE pelo apoio financeiro para a realização deste trabalho. Agradecemos especialmente aos voluntários e seus familiares 


\section{REFERÊNCIAS BIBLIOGRÁFICAS}

1. Obesity: preventing and managing the global epidemic. Report of a WHO consultation. World Health Organ Tech Rep Ser 2000;894:1-253.

2. Siervogel RM, Demerath EW, Schubert C, Remsberg KE, Chumlea WC, Sun S, et al. Puberty and body composition. Horm Res 2003; 60(Suppl 1):36-45.

3. Sistema de Monitoramento de Fatores de Risco e Proteção para Doenças Crônicas Não Transmissíveis por Meio de Inquérito Telefônico. VIGITEL: 2011, Brasil. Disponível em: <http://portalsaude.saude.gov.br/portalsaude/arquivos/pdf/2012/ Abr/10/vigitel_100412.pdf.> [2011 jul 30].

4. Lyon CJ, Law RE, Hsueh W. Minireview: adiposity, inflammation, and therogenesis. Endocrinol 2003;144(6): 2195-200.

5. Yudkin JS. Adipose tissue, insulin action and vascular disease: inflammatory signals. Int J Obes Relat Metab Disord. 2003; 27 Suppl 3:S25-8.

6. Guo SS, Wu W, Chumlea WC, Roche AF. Predicting overweight and obesity in adulthood from body mass index values in childhood and adolescence. Am J Clin Nutr 2002;76(3):653-8.

7. Dyson PA. The therapeutics of lifestyle management on obesity. Diabetes Obes Metab 2010;12:941-6.

8. Parikh T, Stratton G. Influence of intensity of physical activity on adiposity and cardiorespiratory fitness in 5-18 year olds. Sports Med 2011;41(6):477-88.

9. Lazzer S, Boirie Y, Poissonnier C, Petit I, Duche P, Taillardat M, et al. Longitudinal changes in activity patterns, physical capacities, energy expenditure, and body composition in severely obese adolescents during a multidisciplinary weight-reduction program. Int J Obes (Lond) 2005;29(1):37-46.

10. Kuczmarski RJ, Oqden CL, Grummer-Strawn LM, Flegal KM, Guo SS, Wei R. CDC growth charts: United States. Adv Data 2000;8:1-27

11. Tanner JM, Whithouse RH. Clinical Longitudinal standards for height, weight, weight velocity and stages of puberty. Arch Dis Child 1976;51:170-79.

12. Petroski EC, Pires-neto CS, Glaner MF. Biometrica. 1ª ed. São Paulo: Fontoura; 2010. pg.35-39.

13. Slaughter MH, Lohman TG,Boileau RA,Horswill CA,Stillman, RJ, Van Loan MD \&Bemben, DA. Skinfold equations for estimation of body fatness in children and youth. Hum Biol 1988;60,709-23.

14. Wasserman $\mathrm{K}$. The anaerobic threshold measurement to evaluate exercise performance. Am Rev Respir Dis 1984;129(2):S35-40.

15. Prado WL, Siegfried A, Dâmaso AR, Carnier J, Piano A, Siegfried W. Efeito da terapia multidisciplinar de longo prazo sobre a composição corporal de adolescentes internados com obesidade severa. J Pedriat 2009;3(85):243-8.

16. Lazzer S, Lafortuna C, Busti C, Galli R, Tinozzi T, Agosti F. et al. Fat oxidation rate during and after a low- or high-intensity exercise in severely obese Caucasian adolescentes. Eur J Appl Physiol 2009;108:383-91.

17. Lazzer S, Lafortuna C, Busti C, Galli R, Agosti F, Sartorio A. Effects of low- and high-intensity exercise training on body composition and substrate metabolism in obese adolescents. J Endocrinol Invest 2011;34(1):45-52.

18. Slentz CA, Duscha BD, Johnson JL, Ketchum K, Aiken LB, Samsa GP, et al. Effects of the amount of exercise on body weight, body composition, and measures of central obesity: STRRIDE--a randomized controlled study. Arch Intern Med 2000;12164(1):31-9.

19. Chaptini L, Peikin S. Neuroendocrine regulation of food intake. Curr Opin Gastroen 2008;24:223-229.

20. Duscha BD, Slentz CA, Johnson JL, Houmard JA, Bensimhon DR, Knetzger K. et al. Effects of exercise training amount and intensity on peak oxygen consumption in middle-age men and women at risk for cardiovascular disease. Chest 2005;128(4):2788-93. 
21. Nicklas BJ, Wang X, You T, Lyles FM, Demons Jl, Easter L, et al. Effect of exercise intensity on abdominal fat loss during calorie restriction in overweight and obese postmenopausal women: a randomized, controlled trial. Am J Clin Nutr 2009;89:1043-52.

22. Brinkworth GD, Noakes M, Clifton PM. Buckley JD. Effects of a low carbohydrate weight loss diet on exercise capacity and tolerance in obese subjects. Int J Obes 2009;17(10):1916-23.

23. Prado WL, Oyama LM, Lofrano-Prado MC, de Piano A, Stella SG, Nascimento $\mathrm{CM}$, et al. Alterations in downstream mediators involved in central control of eating behavior in obese adolescents submitted to a multidisciplinary therapy. J Adolesc Healt 2011;49(3):300-5.

24. Stella SG, Vilar AP, Lacroix C, Fisberg M, Santos RF, Mello MT. et al. Effects of type of physical exercise and leisure activities on the depression scores of obese Brazilian adolescente girls. Braz J Med Biol Res 2005;38:1683-9.

25. Wong PC, Chia MY, Tsou IY, Wansaicheong GK, Tan B, Wang JC, et al. Effects of a 12-week exercise training programme on aerobic fitness, body composition, blood lipids and c-reactive protein in adolescents with obesity. Ann Acad Med Singapore 2008;37:286-93.

26. Lordelo RA, Mancini MC, Cercato C, Halpern A. Hormonal axes in obesity: cause or effect?. Arq Bras Endocrinol Metabol 2007;51(1):34-41.

27. Thorogood A, Mottillo S, Shimony A, Filion KB, Joseph L, Genest J, et al. Isolated aerobic exercise and weight loss: a systematic review and meta-analysis of randomized controlled trials. Am J Med 2011;124(8):747-55

28. Stiegler P, Adam C. The role of diet and exercise for the maintenance of fat-free mass and resting metabolic rate during weight loss. Sports Med 2006;36:239-62.

29. Hansen D, Dendale P, Berger J, van Loon LJ, Meeusen R. The effects of exercise training on fat-mass loss in obese patients during energy intake restriction. Sports Med 2007;37:31-46.

30. Baldwin J, Snow RJ, Febbraio MA. Effect of training status and relative exercise intensity on physiological responses in men. Med Sci Sports Exerc 2000;32(9):1648-54.
Endereço para correspondência

Wagner Luiz do Prado. Escola Superior de Educação Física (ESEF/UPE)

Rua Arnóbio Marques, 310

Santo Amaro.

Campus Universitário HUOC - ESEF

CEP: 50.100 .130 - Recife- PE. Brasil

E-mail : wagner.prado@pq.cnpq.br 